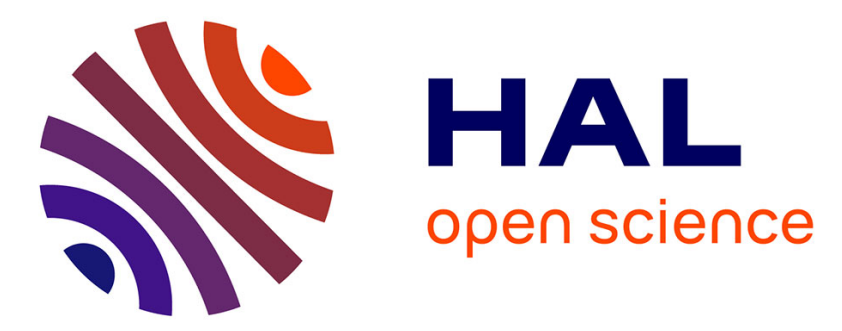

\title{
La bruche du haricot, Acanthoscelides obtectus Say (Coleoptera, Bruchidae), est-elle un danger pour le cowpea, Vigna unguiculata (L.) Walp.?
}

Marc Jarry, Arturo Bonet

\section{- To cite this version:}

Marc Jarry, Arturo Bonet. La bruche du haricot, Acanthoscelides obtectus Say (Coleoptera, Bruchidae), est-elle un danger pour le cowpea, Vigna unguiculata (L.) Walp.?. Agronomie, 1982, 2 (10), pp.963-968. hal-00884337

\section{HAL Id: hal-00884337 \\ https://hal.science/hal-00884337}

Submitted on 1 Jan 1982

HAL is a multi-disciplinary open access archive for the deposit and dissemination of scientific research documents, whether they are published or not. The documents may come from teaching and research institutions in France or abroad, or from public or private research centers.
L'archive ouverte pluridisciplinaire HAL, est destinée au dépôt et à la diffusion de documents scientifiques de niveau recherche, publiés ou non, émanant des établissements d'enseignement et de recherche français ou étrangers, des laboratoires publics ou privés. 


\title{
La bruche du haricot, Acanthoscelides obtectus Say (Coleoptera, Bruchidae), est-elle un danger pour le cowpea, Vigna unguiculata (L.) Walp.?
}

\author{
Marc JARRY \& Arturo BONET $\left(^{*}\right)$ \\ I.B.E.A.S., LA CNRS 340, Université F. Rabelais, Avenue Monge, Parc Grandmont F37200 Tours \\ (*) Instituto de Ecologia, Nuevo Bosque de Chapultepec, Ap. Post. 18-845 Mexico 18 DF, Mexique
}

\section{RÉSUMÉ}

Acanthoscelides obtectus, Vigna unguiculata,

Relations insectes-plantes,

Ponte,

Développement larvaire,

Contamination de graines.
Une fraction de la population d'Acanthoscelides obtectus élevée, depuis plusieurs années sur Phaseolus vulgaris, peut contaminer, dans des conditions expérimentales, des gousses de cowpea, Vigna unguiculata. La présence de graines de cette légumineuse provoque, chez les femelles inséminées, une ponte importante. Un développement larvaire complet dans les graines de cowpea est possible.

La bruche du haricot $A$. obtectus peut donc être un ravageur localement important des stocks de graines de $V$. unguiculata. C'est le cas à Jalapa (Mexique) où l'on a enregistré des contaminations plus ou moins sévères.

\section{SUMMARY}

Acanthoscelides obtectus, Vigna unguiculata, Insect-plant relationships, Egg-laying,

Larval development, Seed contamination.
Can the bean weevil Acanthoscelides obtectus Say (Coleoptera, Bruchidae) attack cowpeas, Vigna unguiculata (L.) Walp.?

Part of a population of Acanthoscelides obtectus reared for several years on Phaseolus vulgaris was able to infest pods of Vigna unguiculata under laboratory conditions. Seeds of $V$. unguiculata induced a high level of egg-laying activity in inseminated females. Complete larval development in seeds of $V$. unguiculata was possible.

$A$. obtectus can therefore be an important local pest of stored $V$. unguiculata seeds. This occurs at Jalapa (Mexico) where fairly severe infestations have been recorded.

\section{INTRODUCTION}

Le cowpea ou niébé, Vigna unguiculata (L.) Walp. est une des légumineuses les plus cultivées en zone tropicale et sub-tropicale pour la production de graines vertes ou sèches et de fourrage (FARIS, 1965).

Callosobruchus maculatus F., qui pond ses æufs sur les gousses ou graines sèches, est considéré comme le danger le plus important pour les graines de Vigna (SouTHGATE, 1978). Bruchidius atrolineatus Pic, qui infeste les gousses vertes (PREVETT, 1961; BOOKER, 1967; SOUTHGATE, 1978) a fait récemment l'objet d'études plus précises (Alzouma, 1981 ; Alzouma \& HUIGNARd, 1981). Zabrotes subfasciatus est également cité à propos de la contamination des graines (DAVIES, 1972; SiNGH \& ALLEN, 1978).

Quelques auteurs signalent Acanthoscelides obtectus Say sur le cowpea (LARSON \& Fisher, 1938 ; STANTON, 1970). Cependant SouthGate (1978), bien qu'il pense qu' $A$. $a b$ tectus est principalement associé à Phaseolus vulga- ris, n'écarte pas les possibilités de développement sur d'autres espèces. Effectivement, $P$. coccineus apparaît comme une espèce avec laquelle les performances reproductrices d'A. obtectus dépassent celles obtenues avec $P$. vulgaris (LEROI \& JARRY, 1981).

En fait, les zones favorables à la culture de Vigna ne favorisent pas $A$. obtectus, très sensible aux températures élevées (Huignard \& BIEMONT, 1974; BIEMONT, 1975).

Cependant, lors d'essais d'introduction de cultures de Vigna dans des zones marginales où sévit la bruche du haricot, la question des relations Vigna/A. obtectus se pose. Ainsi à Jalapa au Mexique $\left(19^{\circ}\right.$ Lat. Nord, $1300 \mathrm{~m}$. d'altitude), nous avons observé des dégâts dus à cet insecte dans les graines stockées.

Cette étude précise l'importance des dégâts causés par A. obtectus dans des stocks de cowpea à Jalapa, l'influence de graines et gousses de $V$. unguiculata sur la fonction reproductrice de la bruche du haricot et les possibilités de développement larvaire dans les graines de cowpea. 


\section{MATÉRIEL ET MÉTHODES}

\section{A. Contamination dans les stocks}

Dix-neuf variétés de $V$. unguiculata et une variété locale de $P$. vulgaris ont été cultivées au Jardin Botanique de Jalapa du 02.05.78 (semis) jusqu'en septembre-octobre 1978 (récoltes) en 4 parcelles pour chaque variété. Après séchage pendant $24 \mathrm{~h}$ à $60^{\circ} \mathrm{C}$, les gousses ont été écossées et les graines ont été stockées dans des sacs en papier (1 sac par parcelle) empilés dans une même pièce où la température minimum n'a jamais été inférieure à $20^{\circ} \mathrm{C}$. L'isolement des graines de chaque parcelle était très relatif, de nombreux sacs n'étant pas étanches et nombreux étaient ceux perforés par des adultes de bruches.

Les attaques ont été estimées par dénombrement des trous d'émergence pour 2000 graines choisies au hasard dans les sacs ( 2 fois 1000 graines pour tester la validité du tirage aléatoire). Pour certaines variétés, il a été possible d'obtenir plusieurs échantillons correspondant à des parcelles différentes. Les dénombrements ont été effectués du 25 janvier au 6 février 1979, soit 3 mois environ après la récolte.

\section{B. Influence de graines et gousses de $V$. unguiculata sur} la reproduction d'Acanthoscelides obtectus

Les expériences ont été réalisées en France à l'I.B.E.A.S.

TABLEAU 1

Estimation des contaminations par A. obtectus des graines stockées de $\mathrm{V}$. unguiculata et $\mathrm{P}$. vulgaris à Jalapa. Estimated infestation of stored seeds of $\mathrm{V}$. unguiculata and $\mathrm{P}$. vulgaris by $\mathrm{A}$. obtectus in Jalapa.

\begin{tabular}{|c|c|c|c|c|c|c|c|c|c|}
\hline \multirow[b]{2}{*}{$\begin{array}{c}\mathrm{N}^{\circ} \\
\text { Variété } \\
\left({ }^{(}\right)\end{array}$} & \multicolumn{3}{|c|}{$1^{\text {er }}$ échantillon } & \multicolumn{3}{|c|}{$2^{\mathrm{e}}$ échantillon } & \multicolumn{3}{|c|}{$3^{e}$ échantillon } \\
\hline & $\begin{array}{l}\text { Nb graines } \\
\text { attaquées } \\
\text { pour } 100 \\
\text { graines }\end{array}$ & $\begin{array}{l}\mathrm{Nb} \text { bruches } \\
\text { pour } 100 \\
\text { graines }\end{array}$ & $\begin{array}{c}\mathrm{Nb} \text { moyen } \\
\text { bruches par } \\
\text { graine } \\
\text { attaquée }\end{array}$ & $\begin{array}{l}\mathrm{Nb} \text { graines } \\
\text { attaquées } \\
\text { pour } 100 \\
\text { graines }\end{array}$ & $\begin{array}{l}\mathrm{Nb} \text { bruches } \\
\text { pour } 100 \\
\text { graines }\end{array}$ & $\begin{array}{l}\text { Nb moyen } \\
\text { bruches par } \\
\text { graine } \\
\text { attaquée }\end{array}$ & $\begin{array}{l}\text { Nb graines } \\
\text { attaquées } \\
\text { pour } 100 \\
\text { graines }\end{array}$ & $\begin{array}{l}\mathrm{Nb} \text { bruches } \\
\text { pour } 100 \\
\text { graines }\end{array}$ & $\begin{array}{c}\mathrm{Nb} \text { moyen } \\
\text { bruches par } \\
\text { graine } \\
\text { attaquée }\end{array}$ \\
\hline 1 & 0 & 0 & - & & & & & & \\
\hline 2 & 0 & 0 & - & & & & & & \\
\hline 3 & 2.0 & 2.95 & 1.47 & 2.0 & 4.9 & 2.45 & & & \\
\hline 4 & 0.1 & 0.2 & 2.00 & 1.4 & 3.0 & 2.14 & & & \\
\hline 5 & 0.25 & 0.6 & 2.40 & 2.25 & 2.95 & 1.31 & & & \\
\hline 6 & 1.25 & 1.45 & 1.16 & 3.35 & 8.1 & 2.41 & & & \\
\hline 7 & & production & très faible, $p$ & is de graines & disponibles & & & & \\
\hline 8 & 6.2 & 13.95 & 2.25 & 1.9 & 2.55 & 1.34 & & & \\
\hline 9 & 1.95 & 4.1 & 2.10 & & & & & & \\
\hline 10 & 7.85 & 12.8 & 1.63 & 19.4 & 52.7 & 2.72 & & & \\
\hline 11 & 1.8 & 2.9 & 1.61 & & & & & & \\
\hline 12 & 13.6 & 27.7 & 2.04 & 5.85 & 9.1 & 1.56 & & & \\
\hline 13 & 0.4 & 0.85 & 2.12 & 1.05 & 1.9 & 1.81 & & & \\
\hline 14 & 0.1 & 0.1 & 1.00 & 2.25 & 3.2 & 1.42 & & & \\
\hline 15 & 1.75 & 2.5 & 1.43 & 0.65 & 1.5 & 2.31 & & & \\
\hline 16 & 0 & 0 & - & & & & & & \\
\hline 17 & 0.1 & 0.1 & 1.00 & 2.5 & 5.5 & 2.20 & & & \\
\hline 18 & 0.9 & 2.3 & 2.56 & 4.3 & 7.65 & 1.78 & & & \\
\hline 19 & 0 & 0 & - & & & & & & \\
\hline 20 & 76.7 & 264.25 & 3.45 & 0.2 & 0.25 & 1.25 & 1.15 & 1.7 & 1.48 \\
\hline
\end{tabular}

(1) $\mathrm{N}^{0} 1$ à $\mathrm{N}^{\prime \prime} 19$ V. unguiculata; N" 20 P. vulgaris. 
avec un matériel biologique totalement différent (bruches élevées depuis plusieurs années sur des graines de $P$. vulgaris, à $27^{\circ} \mathrm{C}$ constant, 75 p. 100 H.R. environ et obscurité). Pour cette souche, la copulation seule ne provoque aucune stimulation de l'ovogenèse (BIEMONT, 1979).

Les gousses et graines de $V$. unguiculata proviennent d'une variété locale du Niger.

Dès leur émergence, les adultes ont été isolés dans des boîtes de Petri avec un abreuvoir contenant du coton imbibé d'une solution de saccharose à 20 p. 100 . Quatre séries d'une cinquantaine de femelles chacune sont constituées simultanément.

Série 1 : femelles vierges

Série 2: femelles vierges +3 graines de Vigna unguiculata Série 3: femelles inséminées +3 graines de Vigna unguiculata

Série 4 : femelles inséminées +3 graines de $P$. vulgaris

Huit jours plus tard, les æufs émis ont été dénombrés ainsi que les ovocytes maintenus en rétention dans les oviductes latéraux des femelles.

Les expériences se sont déroulées dans les conditions de conservation de la souche.

Pour l'étude du comportement de ponte sur gousses, nous n'avons pu effectuer que 2 séries avec 16 femelles inséminées en présence de gousses de $V$. unguiculata ou $P$. vulgaris suspendues par le pédoncule dans des boîtes cylindriques pendant $8 \mathrm{j}$, dans les conditions périodiques suivantes: photopériode $12 \mathrm{~h}-12 \mathrm{~h}$; thermopériode 25 (L)/15 (D) ${ }^{\circ} \mathrm{C}$; hygropériode $60 / 80$ p. $100 \mathrm{H}$.R. Les paramètres retenus ont été la fécondité et la production ovarienne déterminées comme précédemment.

\section{Développement dans les graines de $V$. unguiculata}

Les tests ont porté sur quelques-unes des variétés récoltées à Jalapa selon le protocole indiqué, puis conservées à
l'I.B.E.A.S. à $5^{\circ} \mathrm{C}$ environ. Les larves, fraîchement écloses ont été isolées dans des tubes de verre avec une graine dont le tégument a été, ou non, artificiellement perforé ( 3 trous par cotylédon). La série témoin a été constituée avec des graines de $P$. vulgaris utilisées pour les élevages, récoltées en octobre 1979 et conservées dans les mêmes conditions que celles de Vigna. Tous les tests ont été effectués en février 1981.

\section{RÉSULTATS}

\section{A. Contamination dans les stocks}

Les 2 répétitions du tirage de 1000 graines dans chaque sac ayant toujours donné des résultats comparables, les données ont été regroupées par parcelles. La contamination, estimée 3 mois environ après la récolte et le séchage des graines, est très variable selon les variétés de cowpea, mais aussi selon les parcelles (tabl. 1). L'exemple le plus surprenant est celui des attaques des graines de $P$. vulgaris dont le pourcentage varie de 0,25 à 76,7 p. 100. A la lecture du tableau, l'attaque semble moins sévère pour les graines de $V$. unguiculata que pour celles de $P$. vulgaris.

\section{B. Influence des graines et gousses de $V$. unguiculata sur la reproduction d'A. obtectus}

La présence de graines de $V$. unguiculata ne stimule pas significativement l'ovogenèse des femelles vierges, contrairement aux graines de $P$. vulgaris (POUZAT, 1978a).

Par contre, une grande majorité des femelles inséminées pondent en présence des graines de cowpea et leur production ovarienne est significativement élevée, bien qu'encore inférieure à celles mises en présence de graines de $P$. vulgaris (tabl. 2).

En présence de gousses de V. unguiculata, les femelles

\section{TABLEAU 2}

Ponte, rétention et production ovarienne de femelles d'A. obtectus vierges ou inséminées en présence ou non de graines de $\mathrm{P}$. vulgaris ou V. unguiculata, à l'issue d'une période de 8 jours.

Fecundity, oocyte retention and ovarian production of virgin and inseminated $\mathrm{A}$. obtectus females in the presence or absence of $\mathrm{P}$. vulgaris and $\mathrm{V}$. unguiculata seeds.

\begin{tabular}{|c|c|c|c|c|c|}
\hline & $\begin{array}{l}\text { Nombre } \\
\text { de } \\
\text { femelles }\end{array}$ & $\begin{array}{c}\text { Nombre } \\
\text { de } \\
\text { femelles } \\
\text { pondeuses }\end{array}$ & $\begin{array}{c}\text { Ponte } \\
\text { moyenne }\end{array}$ & $\begin{array}{l}\text { Rétention } \\
\text { moyenne }\end{array}$ & $\begin{array}{l}\text { Production } \\
\text { moyenne }\end{array}$ \\
\hline série 1: femelles vierges & 48 & 0 & 0 & $42.67 \pm 3.94$ & $42.67 \pm 3.94$ \\
\hline $\begin{array}{l}\text { série } 2 \text { : femelles vierges }+ \text { graines de } \\
\quad V . \text { unguiculata }\end{array}$ & 50 & 21 & $4.14 \pm 2.46$ & $41.02 \pm 4.31$ & $45.16 \pm 3.96$ \\
\hline $\begin{array}{l}\text { série } 3 \text { : femelles inséminées + graines de } \\
\quad V . \text { unguiculata }\end{array}$ & 47 & 44 & $56.30 \pm 8.57$ & $17.32 \pm 4.37$ & $73.62 \pm 6.28$ \\
\hline $\begin{array}{l}\text { série } 4 \text { : femelles inséminées + graines de } \\
\quad \text { P. vulgaris }\end{array}$ & 47 & 47 & $76.53 \pm 8.51$ & $17.08 \pm 3.55$ & $93.61 \pm 7.55$ \\
\hline Femelles vierges $\left({ }^{1}\right)$ & 81 & 0 & 0 & 36.23 & $36.23 \pm 2.71$ \\
\hline Femelles vierges + graines de $P$. vulgaris $\left({ }^{1}\right)$ & 40 & $?$ & $15.48 \pm 5.84$ & $46.97 \pm 5.15$ & $62.45 \pm 3.58$ \\
\hline
\end{tabular}


fécondées semblent nettement moins fixées que par les gousses de $P$. vulgaris. En effet, après la découverte de la gousse de $P$. vulgaris, les femelles s'y maintiennent (JARRY, 1980) tandis qu'elles quittent fréquemment celles de $V$. unguiculata. Si 10 femelles sur 16 ont perforé les gousses de cowpea (sans zones préférentielles particulières contrairement à ce qui se passe avec $P$. vulgaris où les perforations sont généralement situées sur la suture ventrale), de nombreux œufs ont été émis hors des gousses et plus particulièrement glissés sous le système de fixation de la gousse (tabl. 3). Plus que la production ovarienne, comparable pour les 2 séries, c'est la variabilité des comportements en présence de gousses de cowpea qui apparaît le phénomène le plus intéressant.

\section{Développement dans les graines de $\boldsymbol{V}$. unguiculata}

La fréquence de pénétration des larves d'A. obtectus dans les graines intactes de quelques variétés de $V$. unguiculata récoltées à Jalapa est toujours très faible (inférieure à 4 p. 100). Même avec les graines de $P$. vulgaris utilisées pour l'élevage, cette fréquence est faible ( 8 p. 100). Dans ces conditions, la mortalité intracotylédonaire est nulle (tabl. 4).

Par contre, avec des graines artificiellement perforées, il y a développement complet pour la moitié au moins des larves de chaque série, y compris pour les variétés n'ayant pas été contaminées à Jalapa (voir tabl. 1). Il n'y a pas de différence significative entre les séries $\left(x^{2}=6,87\right.$ pour 4 d.d.1.). On note toutefois une certaine mortalité larvaire aux $1^{\text {er }}$ et $2^{\mathrm{e}}$ stades (tabl. 5).

\section{DISCUSSION}

Une fraction de la population d'A. obtectus étudiée au laboratoire peut donc se reproduire aux dépens des gousses du cowpea $V$. unguiculata. Ceci n'implique pas forcément que les attaques soient possibles en plein champ, les adultes devant dans ces conditions d'abord découvrir la plante-hôte. Des observations complémentaires seront donc nécessaires pour préciser ce point. De plus la ponte «utile » (c'est-àdire celle émise à l'intérieur des gousses) ne représente, dans les conditions du laboratoire où la phase de découverte est éliminée, qu'un tiers environ de la ponte totale.

Par contre, en présence de graines de $V$. unguiculata, la fécondité des femelles inséminées d'A. obtectus est suffisante pour provoquer des dégâts importants dans les stocks, même si la graine seule ne stimule pas l'ovogenèse chez les femelles vierges, comme c'est le cas avec des graines de $P$. vulgaris.

Le faible taux de pénétration des larves dans les graines de cowpea obtenu dans nos expériences montre une fois de plus l'importance du tégument dans la protection de ces graines, comme c'est le cas chez divers Phaseolus (LEROI \& JARRY, 1981 ; STAMOPOUlOS \& DeSROCHES, 1981), importance qui est également fonction de l'âge des graines et de leur condition de conservation (THIERY, 1981). Ainsi avec des graines plus jeunes obtenues à partir des gousses de

\section{TABLEAU 3}

Ponte, rétention et production ovarienne de femelles d'A. obtectus en présence pendant $8 j$ d'une gousse de $\mathrm{P}$. vulgaris ou $\mathrm{V}$. unguiculata. Fecundity, oocyte retention and ovarian production of $\mathrm{A}$. obtectus females in the presence of a $\mathrm{P}$. vulgaris or $\mathrm{V}$. unguiculata pod for 8 days.

\begin{tabular}{|c|c|c|c|c|c|c|c|c|}
\hline & $\begin{array}{l}\text { Nombre de } \\
\text { femelles }\end{array}$ & $\begin{array}{l}\text { Nombre de } \\
\text { femelles } \\
\text { pondeuses }\end{array}$ & $\begin{array}{l}\text { Nombre de } \\
\text { femelles } \\
\text { ayant percé } \\
\text { la gousse }\end{array}$ & $\begin{array}{l}\text { Nombre de } \\
\text { femelles } \\
\text { avant émis } \\
\text { la totalité } \\
\text { de leur ponte } \\
\text { dans la gousse }\end{array}$ & $\begin{array}{c}\text { Ponte } \\
\text { moyenne }\end{array}$ & $\begin{array}{c}\text { Ponte } \\
\text { moyenne } \\
\text { dans les } \\
\text { gousses }\end{array}$ & $\begin{array}{l}\text { Rétention } \\
\text { moyenne }\end{array}$ & $\begin{array}{c}\text { Production } \\
\text { moyenne }\end{array}$ \\
\hline P. vulgaris & 16 & 16 & 14 & 13 & 70.50 & 63.94 & 4.37 & $74.87 \pm 6.80$ \\
\hline V. unguiculata & 16 & 14 & 10 & 3 & 59.37 & 28.37 & 10.31 & $69.68 \pm 11.62$ \\
\hline
\end{tabular}

TABLEAU 4

Pénétration et développement des larves d'A. obtectus dans des graines intactes de $\mathrm{P}$. vulgaris et de différentes variétés de $\mathrm{V}$. unguiculata. Penetration and development of $\mathrm{A}$. obtectus larvae in intact seeds of $\mathrm{P}$. vulgaris and different varieties of $\mathrm{V}$. unguiculata.

\begin{tabular}{|c|c|c|c|c|c|}
\hline Plante-hôte & $\begin{array}{c}\text { Nombre de } \\
\text { larves } \\
\text { néonates }\end{array}$ & $\begin{array}{c}\text { Nombre de } \\
\text { larves } \\
\text { mortes à } \\
\text { l'extérieur } \\
\text { de la graine }\end{array}$ & $\begin{array}{l}\text { Nombre de } \\
\text { tentatives } \\
\text { visibles } \\
\text { de } \\
\text { perforation }\end{array}$ & $\begin{array}{l}\text { Nombre de } \\
\text { trous de } \\
\text { perforation }\end{array}$ & $\begin{array}{c}\text { Nombre } \\
\text { d'adultes } \\
\text { émergeant }\end{array}$ \\
\hline Vigna unguiculata $\mathrm{N}^{\circ} 1$ & 50 & 50 & 0 & 0 & 0 \\
\hline Vigna unguiculata $\mathrm{N}^{\circ} 8$ & 40 & 40 & 2 & 0 & 0 \\
\hline Vigna unguiculata № 16 & 50 & 48 & 0 & 2 & 2 \\
\hline Vigna unguiculata $\mathrm{N}^{\circ} 19$ & 50 & 48 & 1 & 2 & 2 \\
\hline Phaseolus vulgaris & 50 & 46 & 13 & 4 & 4 \\
\hline
\end{tabular}


TABLEAU 5

Pénétration et développement des larves d'A. obtectus dans des graines perforées de $\mathrm{P}$. vulgaris et de différentes variétés de Vigna unguiculata. Penetration and development of $\mathrm{A}$. obtectus larvae in perforated seeds of $\mathrm{P}$. vulgaris and different varieties of $\mathrm{V}$. unguiculata.

\begin{tabular}{|c|c|c|c|c|c|}
\hline \multirow{2}{*}{ Plante-hôte } & \multirow{2}{*}{$\begin{array}{c}\text { Nombre de } \\
\text { larves } \\
\text { néonates }\end{array}$} & \multirow{2}{*}{$\begin{array}{l}\text { Nombre de } \\
\text { larves mortes } \\
\text { à l'extérieur } \\
\text { de la graine }\end{array}$} & \multicolumn{2}{|c|}{$\begin{array}{c}\text { Mortalité } \\
\text { intracotylédonaire }\end{array}$} & \multirow{2}{*}{$\begin{array}{l}\text { Nombre } \\
\text { d'adultes } \\
\text { émergean }\end{array}$} \\
\hline & & & $\mathbf{L}_{1}$ & $\mathbf{L}_{2}$ & \\
\hline Vigna unguiculata $\mathrm{N}^{\circ} 1$ & 50 & 20 & 7 & 0 & 23 \\
\hline Vigna unguiculata $\mathrm{N}^{\circ} 8$ & 41 & 14 & 6 & 0 & 21 \\
\hline Vigna unguiculata $\mathrm{N}^{\circ} 16$ & 50 & 21 & 6 & 2 & 21 \\
\hline Vigna unguiculata $\mathrm{N}^{\circ} 19$ & 50 & 11 & 6 & 1 & 32 \\
\hline Phaseolus vulgaris & 50 & 18 & 2 & 0 & 30 \\
\hline
\end{tabular}

cowpea utilisées dans nos expériences, le pourcentage de pénétration a pu être estimé à plus de $50 \mathrm{p} .100$.

Par contre les expériences réalisées avec les graines artificiellement perforées, où la mortalité intracotylédonaire est faible, ne mettent pas en évidence l'existence d'une barrière chimique comme on a pu le montrer chez diverses espèces de Phaseolus (LEROI \& JARRY, 1981).

Ces résultats sont difficilement comparables à ceux de JERMY \& SZENTESI (1978) qui obtiennent 100 p. 100 de mortalité avec des graines intactes de $V$. unguiculata. En effet, cette mortalité est donnée globalement pour tous les stades, de la larve L1 aux adultes, sans qu'il soit explicitement indiqué la part due à la mortalité larvaire avant pénétration. D'autre part, les auteurs ne précisent ni l'âge ni les conditions de conservation des graines utilisées. Il n'est donc pas possible de savoir si ces expériences ne mettent pas simplement en évidence le rôle protecteur du tégument, pour des graines âgées par exemple.

Les résultats des contaminations dans les stocks à Jalapa sont difficiles à interpréter. En fait, le protocole expérimental n'avait pas prévu une étude de la contamination par les bruches. Ce n'est qu'a posteriori que nous avons constaté les dégâts dans les stocks et nous avons alors tenté d'en estimer l'importance. Nous resterons donc très prudents dans l'interprétation.

Le point essentiel est que la contamination de stocks de graines de $V$. unguiculata par $A$. obtectus est possible et peut être importante. Or, aucune larve ou nymphe d' $A$. obtectus ne résiste à une température de $60^{\circ} \mathrm{C}$ pendant $24 \mathrm{~h}$, nous l'avons vérifié au laboratoire. On peut alors se poser plusieurs questions :

- Y a-t-il eu contamination à partir d'adultes présents dans les lieux de stockages où sont conservés de nombreux échantillons de graines de diverses légumineuses?

- Toutes les gousses ont-elles subi le même séchage quelle que soit la période de récolte? Ainsi pourraient s'expliquer les différences enregistrées entre les différentes parcelles d'une même variété.

On peut enfin envisager, compte tenu des expériences menées au laboratoire, que seul $P$. vulgaris ait été contaminé en plein champ et que le premier échantillon ait échappé, en partie au moins, au séchage. L'importance des dégâts, pour cet échantillon, serait due à la $2^{\mathrm{e}}$ génération tout à fait possible compte tenu des conditions de stockage (Howe \& Currie, 1964), les adultes de la $1^{\text {re }}$ génération ayant également et de façon variable contaminé les sacs de cowpea. Cette hypothèse est compatible avec les densités d'adultes obtenus (un calcul, trop fastidieux pour être développé ici, montre qu'on peut obtenir à la $1^{\text {re }}$ génération suffisamment d'adultes pour contaminer les autres sacs). Tout ceci reste bien sûr très spéculatif et ne peut être considéré qu'à titre d'hypothèse.

En conclusion, nous insisterons sur la prudence à observer dans ce genre d'interprétation à partir de données de contamination dans des stocks. En particulier, l'absence d'attaques pour certaines variétés n'établit en aucun cas leur " résistance " à tel ou tel bruchidae. Même des expériences contrôlées au laboratoire peuvent être sujettes à caution si l'on ne connaît pas parfaitement l'importance de tous les facteurs qui influencent par exemple la pénétration larvaire dans les graines.

En ce qui concerne la bruche du haricot, $A$. obtectus, ce travail a montré qu'elle peut être localement un ravageur important des graines stockées de $V$. unguiculata. Les possibilités d'adaptation, montrées par une fraction de la population pourtant entretenue au laboratoire depuis plusieurs années sur $P$. vulgaris, indiquent qu' $A$. obtectus, chez qui le polymorphisme est sans aucun doute très important, est un bruchidae capable de s'adapter au cowpea, Vigna unguiculata.

Reçu le 26 mars 1982 Accepté le 7 juillet 1982

\section{REMERCIEMENTS}

Cette étude a été réalisée dans le cadre des accords C.N.R.S.C.O.N.A.C.Y.T. et grâce à l'appui du Secretaria de Educacion Superior e Investigacion de la Secretaria de Educacion Publica (MEXICO).

Nous tenons à remercier également l'I.N.I.R.E.B. qui nous a permis de travailler à Jalapa et Ruben Hernandez JiMENEz qui a collaboré à la collecte des données. 


\section{RÉFÉRENCES BIBLIOGRAPHIQUES}

Alzouma I., 1981. Observations on the ecology of Bruchidius atrolineatus Pic and Callosobruchus maculatus F. in Niger. In " Ecology of Bruchids attacking Legumes (pulses) ", Junk Ed., The Hague, 205-213.

Alzouma I., Huignard J., 1981. Données préliminaires sur la biologie et le comportement de ponte dans la nature de Bruchidius atrolineatus Pic (Coléoptère Bruchidae) dans une zone Sud-Sahélienne au Niger. Oecol. appl. (sous presse).

Biemont J. C., 1975. Influence de modifications de courte durée de la température d'élevage sur la capacité reproductrice de la bruche du haricot (Acanthoscelides obtectus, Coleoptera Bruchidae), in Sterility Principle for Insect Control, I.A.E.A., 186 (6), 279-88.

Biemont J. C., 1979. Influence de la plante-hôte et de la copulation sur la levée de l'inhibition du développement ovarien liée à la rétention des ovocytes chez Acanthoscelides obtectus (Col. Bruchidae). Ann. Soc. entomol. Fr., 15 (1), 93-99.

Booker A. H., 1967. Observation on three Bruchids associated with cowpea in Northern Nigeria. J. stored Prod. Res., 3 : 1-15.

Davies J. C., 1972. A note on the occurrence of Zabrotes subfasciatus on legumes in Uganda. East Afr. agric. for. J., 294-299.

Faris D. G., 1965. The origin and evolution of the cultivated forms of Vigna sinensis. Can. J. Genet. Cytol., 7, 433-452.

Howe R. W., Currie J. E., 1964. Some laboratory observations on the rates of development of several species of Bruchidae breeding in stored pulses. Bull. entomol. Res., 55, 437-477.

Huignard J., Biemont J. C., 1974. Influence d'une augmentation de la température sur la capacité reproductrice des mâles chez la bruche du haricot, Acanthoscelides obtectus. Ann. Zool. Ecol. anim., 6 (4), 561-574.

Jarry M., 1980. Influence de la fixation par la plante-hôte larvaire, Phaseolus vulgaris, sur le comportement de la bruche du haricot, Acanthoscelides obtectus Say. Bull. Int. S.F.E.C.A., 2, 76-86.

Jermy T., Szentesi A., 1978. The role of inhibitory stimuli in the choice of oviposition site by phytophagous insects. Entomol. exp. appl., 24, 258-271.
Larson A. O., Fisher C. K., 1938. The bean weevil and the southern cowpea weevil in California, U.S.D.A. Tech. Bull., 593, $71 \mathrm{pp}$.

Leroi B., Jarry M., 1981. Relations d'Acanthoscelides obtectus Say (Coleoptera : Bruchidae) avec différentes espèces de Phaseolus: influence sur la fécondité et possibilités de développement larvaire. Entomol. exp. appl., 30, 73-82.

Pouzat J., 1978a. Host plant chemosensory influence on oogenesis in the bean weevil, Acanthoscelides obtectus (Coleoptera : Bruchidae). Entomol. exp. appl., 24, 401-408.

Pouzat J., 1978b. La régulation de l'activité reproductrice des femelles de bruche du haricot (Acanthoscelides obtectus Say): influence de quelques facteurs externes (plante-hôte, activité locomotrice). Thèse d'Etat, Université F. Rabelais, Tours, $171 \mathrm{p}$.

Prevett P. F., 1961. Field infestation of cowpea (Vigna unguiculata) pods by beetles of the families Bruchidae and Curculionidae in northern Nigeria. Bull. entomol. Res., 52, 535-54.5.

Singh S. R., Allen D. J., 1980. Pests, diseases, resistance and protection in cowpeas. In "Advances in Legume Science" (Kew 1978), Summerfield \& Butting Ed., 419-443.

Southgate B. J., 1978. The importance of the Bruchidac as pests of grain Legumes, their distribution and control. In "Pests of grain Legumes: Ecology and Control". Singh, Taylor \& Van Emdem Eds. Ac. Press., 219-229.

Stamopoulos D., Desroches P., 1981. Importance of Phaseolus vulgaris seed testa and larval density on Acanthoscelides obtectus Say development; in Ecology of Bruchids attacking Legumes (pulses). Junk ed., The Hague : 165-174.

Stanton W. R., 1970. Les légumineuses à grains en Afrique. F.A.O. Rome, 199 p.

Thiery D., 1981. Etude du comportement des larves néonates d'Acanthoscelides obtectus Say (Coleoptera, Bruchidae) en présence des graines de leur plante-hôte Phaseolus vulgaris $L$. Thèse $3^{\mathrm{e}}$ Cycle, Université F. Rabelais, Tours, 83 p. 